\title{
O papel da ativação comportamental no manejo dos sintomas depressivos
}

\author{
The role of behavioral activation in the management of depressive symptoms \\ El papel de la activación conductual en el manejo de los síntomas depresivos
}

Recebido: 22/11/2021 | Revisado: 01/12/2021 | Aceito: 22/12/2021 | Publicado: 04/01/2022

\author{
Katarime Itanóia Alves \\ ORCID: https://orcid.org/0000-0002-4646-0075 \\ Faculdade Patos de Minas, Brasil \\ E-mail: Katarime@hotmail.com \\ Constance Rezende Bonvicini \\ ORCID: https://orcid.org/0000-0002-6952-625X \\ Faculdade Patos de Minas, Brasil \\ E-mail: constance.bonvicini@faculdadepatosdeminas.edu.br
}

\begin{abstract}
Resumo
A depressão é um transtorno mental complexo e multicausal que impacta severamente a vida do indivíduo que possui seus sintomas. Cada vez mais é possível observar profissionais de diversas áreas da saúde focando seus estudos na busca por compreender tal fenômeno psicológico, seu alcance, suas formas de atuação e as ferramentas de trabalho. A ativação comportamental na depressão é uma ferramenta da psicoterapia, planejada como intervenção para o quadro do paciente, uma vez que se relaciona às emoções do paciente, gera a mudança de humor e comportamento. $\mathrm{O}$ presento trabalho foi construído com o objetivo de descrever essa ferramenta e justifica-se por trabalhar com uma temática que assola milhões de pessoas, discutindo formas mais específicas de se trabalhar com os padrões sintomáticos. A metodologia utilizada foi a revisão narrativa da literatura, através da análise de obras já publicadas sobre o assunto. Assim, pode-se concluir que a ativação atua no processo de tratamento reduzindo a esquiva e a ruminação aplicando-se técnicas como: registro diário, prazeres que o paciente identifica, atividades com os horários, manejo de contingências situacionais, ensaio verbal das tarefas propostas e técnicas de relaxamento, ou seja, todos os passos com o paciente ativo, de fora para dentro.
\end{abstract}

Palavras-chave: Ativação comportamental; Terapia cognitivo-comportamental; Depressão; Transtorno depressivo maior.

\begin{abstract}
Depression is a complex and multi-causal mental disorder that roundly impacts the individual's life who has its symptoms. It is becoming more possible to observe professionals from different areas of health focusing their studies on the seek to understand this psychological phenomenon, its reach, its ways of acting and working tools. Behavioral activation in depression is a psychotherapy tool, planned as an intervention for the patient's condition, once it is related to the patient's emotions, bringing forth a change in mood and behavior. The present work was developed aiming to describe this tool and it is justified for working with a theme that affects millions of people, discussing more specific ways of working with these symptomatic patterns. The methodology used was the narrative literature review, through the analysis of works that have already been published on the subject. Therefore, it can be concluded that activation acts in the treatment process, reducing avoidance and rumination with techniques: record diary, pleasures that the patient identifies, recording of activities with schedules, handling situational contingencies, verbal rehearsal of proposed tasks and techniques of relaxation, that is, all the steps with the active patient from out to the inside.
\end{abstract}

Keywords: Behavioral activation; Cognitive behavioral therapy; Depression; Major depressive disorder.

\section{Resumen}

La depresión es un trastorno mental complejo y multicausal que afecta gravemente la vida de la persona que tiene sus síntomas. Cada vez es más posible observar a profesionales de diferentes áreas de la salud centrando sus estudios en la búsqueda por comprender este fenómeno psicológico, su alcance, sus formas de actuar y herramientas de trabajo. La activación conductual en la depresión es una herramienta de psicoterapia, planificada como una intervención para la condición del paciente, ya que se relaciona con las emociones del paciente, generando un cambio de humor y comportamiento. El presente trabajo se construyó con el objetivo de describir esta herramienta y se justifica por trabajar con un tema que afecta a millones de personas, discutiendo formas más específicas de trabajar con estos patrones sintomáticos. La metodología utilizada fue una revisión de literatura, mediante el análisis de trabajos ya publicados sobre el tema. Así, se puede concluir que la activación actúa en el proceso de tratamiento, reduciendo la evitación y rumia con técnicas de registro diario, placeres que el paciente identifica, registro de actividades con horarios, manejo de contingencias situacionales, ensayo verbal de tareas propuestas y técnicas de relajación, que es decir, todos los pasos con el paciente activo, desde el exterior hacia el interior.

Palabras clave: Activación conductual; Terapia de conducta cognitiva; Depresión; Trastorno depresivo mayor. 


\section{Introdução}

Os transtornos mentais são motivo de grande preocupação mundial, tanto ao que tange as políticas públicas, que permeiam a saúde mental individual, quanto a coletiva. A depressão é um transtorno mental complexo, multicausal e que impacta severamente a vida da família e do indivíduo em que se configuram os sintomas. Cada vez mais é possível observar profissionais de diversas áreas da saúde focando seus estudos na busca por compreender esse fenômeno psicológico, seu alcance, suas formas de atuação e as ferramentas de trabalho.

Cardoso (2017) aponta que a depressão é um transtorno de humor, no qual o indivíduo se apresenta frequentemente entristecido, desanimado, com ausência da vontade de fazer o que gosta. Em geral, as atividades se tornam sem sentido e desprovidas de prazer, desenvolvendo um retraimento social gradual e um padrão de esquiva para evitar problemas. O mesmo autor acrescenta que dentre outros sintomas relevantes, ocorrem alterações do peso corporal (ganho ou perda de peso significativo $-5 \%$ ), prejuízos no sono (insônia ou hipersonia), agitação ou retardo psicomotor, sentimento de inutilidade ou culpa, baixa capacidade de concentração ou tomada de decisão e/ ou ideação suicida (Associação Americana de Psicologia [APA], 2004; Organização Mundial de Saúde [OMS], 1993).

Segundo Kerbauy (1983), na Terapia Cognitiva Comportamental (TCC), os pensamentos das pessoas são o centro da análise, uma vez que em decorrência desses pensamentos, as emoções e situações vivenciadas pelo indivíduo podem provocar e causar distúrbios comportamentais. Guimarães et al. (2017) aponta que o modelo é conhecido por sua metodologia breve, de sessões estruturadas e focadas no "aqui e agora", a partir de um processo de engajamento ativo do paciente durante as sessões.

Knapp e Beck (2008) apontam que a depressão se manifesta no indivíduo de forma a induzi-lo a ter uma percepção alterada das situações. No cérebro parece haver ativação de memórias emocionais e sensitivas que conotam representação negativa de si mesmo, dos outros e do futuro/ mundo, conhecida como tríade cognitiva. Dentre as técnicas mais utilizadas no tratamento da depressão, os autores destacam aquelas relacionadas ao automonitoramento, à restruturação cognitiva, à técnicas de enfrentamento, ao treino de habilidades sociais e à resolução de problemas. Wright et al. (2008) destacam que a ativação comportamental, a programação de atividades, o planejamento de tarefas graduais e o ensaio comportamental são também intervenções comportamentais que podem ajudar no tratamento da depressão.

A ativação comportamental na depressão é uma ferramenta da psicoterapia, planejada como uma intervenção para ajudar na depressão, uma vez que ela está relacionada com as emoções do paciente, gerando mudanças no humor e no comportamento. Sua atuação compreende atividades para enfrentamento de esquivas rotineiras através do uso de diversas técnicas, dentre elas a resolução de problemas, visando auxiliar o paciente que tem dificuldades em se movimentar e enfrentar suas dificuldades (Santos, 2017).

O presente texto foi construído com o objetivo de descrever tal ferramenta e justifica-se por trabalhar com uma temática que assola milhões de pessoas e discutir formas específicas de se trabalhar com esses padrões sintomáticos. O texto está organizado em três seções: a primeira traz a apresentação da depressão e os padrões sintomáticos; a segunda seção foca na descrição do conceito e das características da ativação comportamental; e a terceira seção destina-se à avaliação do manejo clínico dos sintomas depressivos através do uso da ativação comportamental como ferramenta de trabalho do terapeuta cognitivo-comportamental.

\section{Metodologia}

O presente estudo caracteriza-se como uma revisão narrativa da literatura. Segundo Patrícia de Oliveira Bastos (2021) a revisão narrativa é aquela que agrega conhecimentos, de forma enriquecedora para discutir e esclarecer as dúvidas sobre determinada temática em questão. Fazendo ressurgir e desenvolver outras ideias, possuindo base e ligações com outros 
conhecimentos, criando-se outras teorias diferentes sobre cada temática. Possui característica importante do ponto de vista metodológico:

As revisões narrativas não necessitam ficar atreladas a informar fontes de informações utilizadas, a metodologia para a busca das referências, nem os critérios utilizados na avaliação e seleção dos trabalhos consultados (Rother et.al., 2007).

O presente estudo foi realizado a partir da busca de materiais em bibliotecas físicas e digitais, que incluem Scielo, google acadêmico entre outros. Não foi realizado um recorte temporal de publicações por considerar que existem obras clássicas relevantes para a temática em estudo. Para a busca de material utilizou-se obras em português e inglês que incluem livros, artigos, monografia e tese recuperados a partir da utilização das seguintes palavras-chave: ativação comportamental, terapia cognitiva comportamental, depressão maior.

\section{Depressão}

A depressão se caracteriza como um transtorno de humor, no qual são observadas alterações em quatro áreas sintomáticas, sendo elas: sintomas emocionais (tristeza, perda de prazer, sensação de culpa); sintomas cognitivos (visão negativa de si mesmo, desesperança, enfraquecimento da concentração e memória); sintomas motivacionais (passividade, falta de iniciativa e de persistência); e sintomas físicos (mudança do apetite e sono, fadiga, aumento de dores e mal-estar nas atividades) (Rufino et al., 2018).

Gonçalves et al. (2018) destacam que de acordo com o DSM-V é possível classificar a depressão em três níveis: depressão menor, depressão distimia e depressão maior. Dentre os critérios diagnósticos temos: anedonia (perda de prazer pelas atividades), sentimento de inutilidade ou de culpa frequente, dificuldade de concentração, fadiga ou perda de energia, distúrbio do sono (insônia ou hipersonia) diariamente, problemas psicomotores (agitação ou retardo psicomotor), perda ou ganho de peso significativo, ideias recorrentes à morte e suicídio. A distinção desses níveis está relacionada a apresentação dos sintomas. Na depressão menor é necessário apresentar de dois a quatro sintomas por duas ou mais semanas, incluindo estado deprimido ou anedonia. Já na distimia são necessários três ou quatro sintomas, incluindo estado deprimido, durante no mínimo dois anos. Para a depressão maior são necessários cinco ou mais sintomas por duas semanas ou mais, incluindo estado deprimido ou anedonia.

Reporta-se por um alerta da OMS que a depressão se configura como um relevante problema de saúde pública, sendo considerado um transtorno grave, recorrente e que leva o indivíduo a uma perda significativa de sua funcionalidade. Os dados mundiais estimam que mais de 300 milhões de pessoas vivam com depressão, o que retrata um aumento de mais de $18 \%$ entre o período de 2005 e 2015 (Lima et al., 2020).

Especificamente no continente americano, o número de pessoas afetadas gira em torno de 50 milhões, representando a quarta doença que mais causa incapacidade por consequência de perda na qualidade de vida (Gonçalves et al., 2018). No Brasil, a depressão atinge 11,5 milhões de pessoas (5,8\% da população).

Leahy (2015) aponta que a depressão alcança qualquer indivíduo, independentemente de renda, gênero, educação, sucesso, raça, beleza, isto é, ela não se atém a obstáculos. O autor enfatiza que a depressão está relacionada à química do cérebro humano, destacando que diferentes níveis de serotonina, noradrenalina e outras substâncias atuam para tornar o indivíduo mais predisposto ao desenvolvimento de um episódio depressivo. Além disso, não se deve descartar entre os fatores que influenciam nas probabilidades de um episódio, todo o processo de aprendizagem do indivíduo, desde a sua criação até experiências posteriores na vida.

Shatzberg (2009 citado por Gomes, 2018) reporta que são muitas as teorias que buscam compreender as origens da 
depressão, sendo as teorias neurocientíficas as que atestam para a existência de déficits funcionais de monoaminas (para casos de sintomas depressivos) e/ou excesso dos neurotransmissores (para casos de sintomas maníacos), considerada uma das principais pelos cientistas. Leahy (2015) reforça essa visão pontuando que a noradrenalina, a serotonina e a dopamina fazem parte da classe das monoaminas e são três grupos de neurotransmissores que devem ser consideradas relevantes ao afetar a depressão.

Considerando que mudanças transitórias de humor são experimentadas por todos os humanos, sabe-se que a depressão intensa pode seguir uma experiência traumática ou a perda de um ente querido, ou ainda ser um sintoma de muitas condições médicas, incluindo acidente vascular cerebral, doença de Cushing, hipotireoidismo, esclerose múltipla, doença de Huntington e doença de Parkinson. Da mesma forma, a depressão também pode ser precipitada por agentes farmacológicos como a reserpina, que esgota a dopamina nas sinapses. O suporte adicional para as bases biológicas do transtorno depressivo maior (TDM) deriva de alguns dos principais sintomas do próprio transtorno, que incluem desregulações dos ritmos circadianos, processamento cognitivo e funcionamento apetitivo e psicomotor (Belmaker \& Agam, 2008).

As monoaminas têm sido o foco principal das teorias etiológicas anteriores do TDM. Embora a hipótese de depleção de monoamina agora pareça ser uma visão simplificada da fisiopatologia do TDM, deve-se reconhecer o significado histórico da hipótese, na medida em que ajudou a desenvolver vários novos medicamentos antidepressivos, cada um possuindo a capacidade de afetar o sistema de monoaminas em uma maneira relativamente seletiva. Em particular, o papel putativo da serotonina no TDM tem sido amplamente estudado, em parte por causa dos amplos efeitos terapêuticos na depressão da droga, como os inibidores seletivos da recaptação da serotonina (Otte et al., 2016).

Uma vez que os efeitos benéficos dos medicamentos antidepressivos são observados após várias semanas, os pesquisadores propuseram que esses tratamentos requerem mudanças nos fatores de transcrição e na expressão gênica (Duman et al., 1997). Uma hipótese é que as drogas antidepressivas elevam os níveis de fator neurotrófico derivado do cérebro (BDNF) por meio de CAMP e efeitos mediados por CREB da transcrição da mensagem do BDNF (Duman et al., 1997). Em última análise, entretanto, os antidepressivos podem alterar a função sináptica e a atividade do circuito, como foi postulado que ocorre no vício (Berke \& Hyman, 2000). O TDM é frequentemente recorrente ou crônico e as evidências sugerem que apesar dos fatores genéticos influenciarem parcialmente o risco geral de doença, eles também influenciam a sensibilidade dos indivíduos aos efeitos depressogênicos da adversidade ambiental. $\mathrm{O}$ tratamento com antidepressivos ou certas formas de psicoterapia é bastante eficaz, mas uma proporção substancial de pacientes não responde adequadamente, exigindo intervenções subsequentes.

Para análise de cada caso no diagnóstico e tratamento da depressão, as comorbidades estão relacionadas e devem ser levadas em conta pelas condições do paciente, como por exemplo, a existência de outros transtornos, como o de ansiedade, o transtorno obsessivo-compulsivo, o abuso de substâncias, os transtornos da personalidade, o transtorno de pânico, a fobia social (Wright et al., 2008). De acordo com a comorbidade, a depressão dobra o risco de evoluir para um quadro ruim, ou seja, um prognóstico negativo, podendo gerar transtornos como o de personalidade borderline, transtorno de personalidade obsessiva compulsiva, transtorno de personalidade paranoide, transtorno de personalidade esquizoide, transtorno de personalidade evitativa e transtorno de personalidade antissocial (Dalgalarondo, 2019).

Lopes (2005) pontua que a forma como a depressão se apresenta em cada indivíduo é diferente, uma vez que são diversas as formas de desencadeamento dos sintomas e isso torna a tarefa de padronização de sintomas complexa. Leahy (2015) sinaliza que a depressão pode assumir a forma de pensamentos autocríticos, de perturbação do sono, de indecisão, de irritabilidade, de baixa energia, de tristeza, de retraimento e de outros sintomas.

Os sentimentos e os comportamentos dos indivíduos segundo Kanapp (2008), a forma como eles os interpreta e visualiza o mundo se processa de acordo com os seus pensamentos e crenças. Beck explica que a causa da depressão seriam as 
crenças disfuncionais, envolvendo às vezes a ideia de não ser amado ou aceitável e causando pensamentos negativos, mudando a percepção da realidade e refletindo no comportamento do indivíduo através da manifestação de sintomas (Medeiros, 2010). As ideias de culpa estão associadas a pensamentos de autodepreciação, desesperança (pessimismo), ideias de fracasso, pensamentos de autocompaixão, ideias suicidas, hipocondrias, pensamentos e desejo de morte e outros. O modelo cognitivo, o humor e os comportamentos negativos seriam resultados de pensamentos e crenças distorcidas, envolvendo tanto o surgimento, quanto a manutenção da depressão.

Wright et al. (2008) discorrem que existem diversos métodos cognitivo-comportamentais para o tratamento da depressão, incluindo intervenções específicas que foram desenvolvidas para reverter níveis diminuídos de atividade, depleção da energia, piora da anedonia e capacidade reduzida de concluir tarefas ou resolver problemas.

Cardoso (2017) aponta que a terapia cognitivo-comportamental consiste no desenvolvimento e aplicação de estratégias que favoreçam mudanças comportamentais e cognitivas. Dessa forma, os comportamentos que são focos de alteração é que determinam a escolha das estratégias interventivas a serem utilizadas. A mesma autora destaca ainda outras formas de tratamento, como a terapia comportamental (ou analítico comportamental), a terapia de aceitação e compromisso, a psicoterapia analítico funcional e a ativação comportamental.

\section{Conhecendo o Papel da Ativação Comportamental na Intervenção com Pacientes Depressivos}

A fim de se delimitar conceitualmente e estabelecer um foco para o presente artigo, define-se a seguir o que é a Ativação Comportamental. A Behavior Activation (BA) ou Ativação Comportamental tem por objetivo aumentar o repertório comportamental e promover atividades de resolução de problemas para que o indivíduo possa entrar em contato com a contingência de reforçamento positivo (Cardoso, 2017).

A definição da ativação comportamental é estabelecida pelo manual de terapia cognitiva no tratamento da depressão, chamada de técnica comportamental (Abreu, 2020). A utilização da BA com clientes gravemente depressivos objetiva restaurar um funcionamento mínimo para que eles consigam responder às técnicas cognitivas direcionadas à mudança de pensamentos (CarneiroI, 2016). A ativação comportamental é desenvolvida por psicólogos cognitivos comportamentais, buscando resultados de efetividade similares à da TCC tradicional, sem intenção de técnicas cognitivas ou da necessidade de anos de treinamento intensivo. As bases de princípios são do condicionamento operante, sempre considerando a depressão a partir do contexto da pessoa e entendida de acordo com suas ações e comportamentos relacionados ao ambiente.

Para CarneiroI (2016), a atuação da depressão é o foco da ativação, sendo seus processos o que proporciona a redução da esquiva e da ruminação, através da observação dos problemas, das rotinas para a aplicação das estratégias de enfrentamento. Dentre tais estratégias estão o diário de maestria e prazer, o registro das atividades com horários, o manejo de contingências situacionais, o ensaio comportamental, a modelagem de estratégias de ativação, o ensaio verbal das tarefas propostas e as técnicas de relaxamento.

O papel do paciente é analisar as consequências não intencionais de suas maneiras de respostas, encontrar as razões do passado e tentar resolver os problemas insolúveis. Os pacientes mostram que as suas formas de enfrentamento são mais retraídas, pois evitam as suas atividades normais e a interação social. Relatam que a forma de se relacionar leva a depressão mais profunda e a perda de experiências da vida, que seriam o que traria a satisfação ou o prazer. A forma de agir com o ambiente e as outras pessoas é que pode agravar a depressão (Ferreira, 2020).

A ativação comportamental é baseada no entendimento bem pesquisado de que a depressão muitas vezes impede o paciente de fazer as coisas que trazem prazer e significado às suas vidas. Esta "espiral descendente" faz com que o paciente se sinta ainda pior. Na ativação comportamental, trabalha-se para reverter esse ciclo usando as próprias ações e escolhas do paciente (Manos et al., 2011). 
Dimidjian et al., (2014) compreendem que a ativação comportamental envolve variados seguintes aspectos, como a compreensão dos "ciclos viciosos" da depressão, acompanhando as atividades diárias do paciente e identificando os objetivos e valores desse; a construção de uma espiral ascendente de motivação e energia por meio do prazer e da maestria; o agendamento das atividades agradáveis e significativas, solucionando os problemas em torno de barreiras potenciais à ativação, reduzindo a evasão ou esquiva. Foca-se no trabalho em equipe para fazer um progresso gradual, sistemático e sustentado, sendo usadas as atribuições entre as sessões (Dimidjian et al., 2014).

Outro aspecto importante é deixar o paciente ciente de seu humor, de suas emoções e comportamentos, monitorando essas emoções de forma bastante consciente. Para saber o que fazer para resolver um problema, primeiro é necessário que tanto terapeuta, quanto paciente, entendam de verdade o que está acontecendo. O monitoramento de atividades é a primeira etapa da ativação comportamental. É importante saber exatamente o que o paciente está fazendo ao longo do dia e como isso corresponde ao seu humor. Embora não seja possível "curar" a depressão apenas observando esses aspectos, esse pode ser um passo para que o paciente possa se sentir melhor, entendendo quais comportamentos o ajudam nisso, quais mantêm a depressão como está e quais o faz se sentir pior (Stein et al., 2021).

A próxima etapa da ativação comportamental é a determinação dos comportamentos em que os pacientes devem se concentrar para melhorar seu humor. Embora alguns comportamentos, como exercícios e meditação, possam ser usados imediatamente para melhorar o humor de forma direta, muitos dos comportamentos que provavelmente ajudarão o paciente depressivo são aqueles que se alinham com as coisas que eles realmente gostam de fazer ou são importantes para os mesmos. Uma exploração dos valores, do prazer e do domínio descreve muitas das "coisas" que fazem a vida valer a pena para aquele paciente em especial. Esse processo pode auxiliar a definir metas tangíveis que movam os pacientes em direção aquilo que é mais importante para eles (Barraca et al., 2011).

Valores são as coisas significativas na vida do paciente, as mais importantes para o indivíduo. Cada pessoa tem valores diferentes e, para cada pessoa, eles podem mudar com o tempo. São como uma bússola que aponta e norteia para a direção que a pessoa quer ir ou levar a sua vida. O prazer, por sua vez, envolve atividades ou brincadeiras que despertam no indivíduo sensações agradáveis e de satisfação, como hobbies, jogos, estar na natureza ou com um bom amigo (Barraca et al., 2011).

O domínio envolve atividades, como trabalho ou esportes, que envolvem o desenvolvimento de habilidades, de ações que os pacientes são capazes de realizar e terem uma sensação de domínio sobre seu ambiente. Quando desfrutados com moderação e bem diversificados com outras atividades, podem aumentar as emoções positivas e melhorar a forma como os pacientes sentem sobre si mesmos e o ambiente em que estão. Além disso, essas atividades podem fazer o paciente se sentir mais criativo à medida que aprende a dominar certas habilidades, aumentando as possibilidades de diversão (Barraca et al., 2011).

Metas e objetivos delineiam os passos que os indivíduos darão para experimentar suas vidas com mais plenitude, e muitas vezes a pessoa com depressão pode não sentir mais vontade de manejar essas metas e objetivos. Assim, o fato de ter objetivos fornece ao paciente metas para auxiliá-lo a ter mais prazer, domínio e comportamento orientado por valores (Hopko et al., 2011). Para se sentir mais consistentemente engajado e feliz no mundo, geralmente é melhor encontrar um equilíbrio de objetivos centrados em valores, no prazer e no domínio. A aparência desse equilíbrio para cada paciente será única.

A ativação comportamental pode ser um desafio para o paciente, por isso é necessário que haja comunicação e compartilhamento das etapas entre paciente e terapeuta. É comum que eles encontrem obstáculos durante esse processo e tenham momentos em que possam querer desistir. Pode-se dizer que a única barreira para a melhoria é a desistência integral, pois se em contrapartida, o paciente continue a aprender sobre seu curso de vida e consiga seguir com o tratamento é provável que as coisas melhorem para o mesmo (Barraca et al., 2011). 
Exemplos de estudos apontam a efetividade da ativação comportamental através de pesquisa que contaram com terapeutas cognitivos comportamentais. A análise focou na comparação dos resultados das três condições de tratamento em dois grupos estratificados: os participantes com depressão leve e os participantes com depressão de moderada à severa. Os resultados na melhora dos participantes que receberam BA foram os mesmos dos que receberam a medicação, mesmo entre os mais severamente depressivos. Pacientes designados para BA permaneceram mais tempo em tratamento se comparado com os que receberam medicação. Conclui-se que a BA foi superior à terapia cognitiva no tratamento dos pacientes severos e que não houve diferença significativa entre as três modalidades de tratamento no caso de paciente com depressão leve (Abreu, 2020).

Os resultados de um estudo de seguimento de dois anos indicaram que os benefícios da BA, semelhantemente aos da terapia cognitiva, ajudaram a prevenir recaídas e episódios futuros de depressão, sendo comparáveis aos índices dos pacientes que suspenderam a medicação (Dobson et al., 2008). Já os pacientes designados para a medicação tiveram maiores taxas de recaídas ao longo do segundo ano de tratamento quando comparadas as dos pacientes submetidos anteriormente a BA ou a terapia cognitiva (Dimidjian et al., 2006; Dobson et al., 2008).

Por fim, retratando as possíveis limitações da ferramenta, quanto às intervenções, pontua-se que nenhuma quantidade de ativação comportamental individual, exposição ou treinamento de habilidades poderá mudar a história do cliente. O que se verifica é que esses pacientes ficam mais bem equipados para mudar suas circunstâncias, tanto as presentes, quanto as futuras. Se eles forem capazes de minimizar os agravamentos, de reduzirem sintomas e comportamentos problemáticos, bem como aumentar o nível de suas habilidades em áreas diferentes, serão também mais capazes de melhorar suas vidas e de exercer uma influência positiva sobre as pessoas que os cercam (Dobson, 2010).

\section{Considerações Finais}

A depressão é um transtorno cada vez mais prevalente no mundo. A cada dia que passa vários casos vêm surgindo, despertando a busca pelo entendimento sobre os tratamentos, enfatizando-se nesse artigo, o papel da ativação comportamental da abordagem da Terapia Cognitivo- Comportamental. Nesse estudo de revisão fora descrito o uso da ativação comportamental como ferramenta de trabalho do psicólogo da TCC. A hipótese de que a ativação comportamental tenha eficácia no tratamento da depressão e que os pacientes possuem poucas recaídas se em comparação com os tratamentos somente medicamentosos é

comprovada. É evidente que de acordo com o quadro depressivo do paciente se torna necessária a medicação, através da reflexão de um psiquiatra junto ao psicoterapeuta, que busquem pela melhora dos sintomas em geral, através da combinação multidisciplinar. Portanto, o papel da ativação comportamental no manejo dos sintomas depressivo é muito enriquecedor, pois trabalha as emoções, como a tristeza, a perda de prazer, a sensação de culpa; os sintomas cognitivos, como a visão negativa de si mesmo, a desesperança, o enfraquecimento da concentração e da memória; sintomas motivacionais como a passividade, a falta de iniciativa e de persistência; e sintomas físicos, mudança do apetite e sono, fadiga, aumento de dores e mal-estar nas atividades em geral.

A ativação atua nos processos cognitivos reduzindo a esquiva e a ruminação através da observação dos problemas, das rotinas e de estratégias de enfrentamento, contribuindo assim para uma melhora do quadro da doença e para uma melhor qualidade de vida, através do autoconhecimento e do olhar para si.

Sugere-se que sejam realizados novos estudos para trazer à tona novas ideias, novos pensamentos ao debate com o auxílio de periódicos da área que fazem um estudo minucioso e cientificamente relevante. Ampliando a discussão do tema para a aplicação dessa intervenção em idosos e crianças, para que no futuro, o presente trabalho possa ser colocado na mesa dos especialistas que possam vislumbrar demandas específicas voltadas para esse público. E que essas pesquisas possa servir, também, como inspiração a novos estudos que queiram trazer uma nova abordagem e possam utilizar este trabalho como referencial teórico. 


\section{Agradecimentos}

Agradeço a Deus e a todos os que me deram suporte emocional e cognitivo para execução desse trabalho.

\section{Referências}

Abreu, J. H. P. R. (2020). Ativação Comportamental Na Depressão. Manole.

Associação Americana de Psicologia [APA] (2004). https://www.apa.org/pubs.

Barraca, J., Pérez-Álvarez, M., \& Bleda, J. H. L. (2011). Avoidance and activation as keys to depression: Adaptation of the Behavioral Activation for Depression Scale in a Spanish sample. The Spanish journal of psychology, 14(2), 998-1009.

Belmaker, R. H., \& Agam, G. (2008). Major depressive disorder. New England Journal of Medicine, 358(1), 55-68.

Berke, J. D., \& Hyman, S. E. (2000). Addiction, dopamine, and the molecular mechanisms of memory. Neuron, $25(3), 515-532$.

Cardoso, L. R. D. (2017). Psicoterapias comportamentais no tratamento da depressão. Psicologia Argumento, 29(67), 479-489.

Carneirol, A. M., \& Keith, (2016). Tratamento cognitivo-comportamental para depressão maior: uma revisão narrativa. Revista Brasileira de Terapias Cognitivas, Porto Alegre, 12(1), 42-49.

Dalgalarondo, P. (2019). Psicopatologia e semiologia dos transtornos mentais. Artmed.

Dobson, D. K. S. (2010). A Terapia Cognitivo-Comportamental Baseada em Evidências. Artmed.

Dimidjian, S., Martell, C. R., Herman-Dunn, R., \& Hubley, S. (2014). Behavioral activation for depression. In D. H. Barlow (Ed.), Clinical handbook of psychological disorders: a step-by-step treatment manual. The Guilford Press, 353-393.

Dobson, K. S., Hollon, S. D., Dimidjian, S., Schmaling, K. B., Kohlenberg, R. J., Gallop, R. J. et al. (2008). Randomized trial of behavioral activation, cognitive therapy, and antidepressant medication in the prevention of relapse and recurrence in major depression. Journal of Consulting and Clinical Psychology, 76(3), 468-477.

Duman, R. S., Heninger, G. R., \& Nestler, E. J. (1997). A molecular and cellular theory of depression. Archives of general psychiatry, 54(7), 597-606.

Ferreira, A., \& Silva, P. (2020). Modelos tradicionais de tratamento da depressão na análise do comportamento. Revista Terra \& Cultura: Cadernos de Ensino e Pesquisa, 36(70), 37-46.

Gonçalves, A. M. C., Teixeira, M. T. B., Gama, J. R. A., Azevedo, C. S. L., Silva, G. A. Gamarraet, C. Justina G. et al. (2018). Prevalência de depressão e fatores associados em mulheres atendidas pela estratégia de saúde da família. Jornal Brasileiro de Psiquiatria, 67(2), $101-109$.

Guimarães, L. C., Werpp, M., \& Santos, L. A. (2017). Eficácia de intervenções comportamentais no tratamento de pacientes com diagnostico de transtorno depressivo maior. Psicologia.pt, p. 8. https://www.psicologia.pt/artigos/ver_artigo.php?eficacia-de-intervencoes-comportamentais-no-tratamento-de-pacientescom-diagnostico-de-transtorno-depressivo-maior\&codigo=A1082

Hopko, D. R., Magidson, J. F., \& Lejuez, C. W. (2011). Treatment failure in behavior therapy: focus on behavioral activation for depression. Journal of clinical psychology, 67(11), 1106-1116.

Kerbauy, R. R. (1983). Terapia comportamental cognitiva: uma comparação entre perspectivas. Psicologia: Ciência e Profissão, 3(2), 11-23.

Knapp, P. \& Beck, A. T. (2008). Fundamentos, modelos conceituais, aplicações e pesquisa da terapia cognitiva. Brazilian Journal of Psychiatry, São Paulo, 30(2), 54-64.

Leahy, R. L. (2015). Vença a depressão antes que ela vença você. Trad. Sandra aria Mallmann da Rosa; Revisão: Bernard P. Rangé, Artmed.

Lima, L. G. B., Santos, A. E., Santos, B. L. C., \& Pedrão, L. J. (2020). Características de usuários com diagnóstico de Transtorno Depressivo atendidos em um Centro de Atenção Psicossocial. SMAD. Revista eletrônica saúde mental álcool e drogas, 16(1), 1-9.

Lopes, J. P. (2005). Depressão: uma doença da contemporaneidade - uma visão analítico- comportamental. Monografia de Graduação em Psicologia, Faculdade de Ciências da Educação e Saúde, Centro Universitário de Brasília, Brasília.

Manos, R. C., Kanter, J. W., \& Luo, W. (2011). The behavioral activation for depression scale-short form: development and validation. Behavior therapy, Chicago, 42(4), 726-739.

Medeiros, H. L. V. (2010) Distorções do pensamento em pacientes deprimidos. Jornal Brasileiro de Psiquiatria, 59(1), 28-33.

Organização Mundial de Saúde [OMS]. Relatório Mundial de Saúde. (1993). https://www.who.int/eportuguese/publications/WHR2010.pdf.

Otte, C., Gold, S. M., Penninx, B. W., Pariante, C. M., Etkin, A., Fava, M. et al. (2016). Major depressive disorder. Nature reviews Disease primers, 2(1), 120 .

Patrícia O. B., J. J. (2021 de 07 de 26). Atuação do enfermeiro brasileio no ambiente escolar: Revisão narrativa. Research, Society and Development, 8. 
Research, Society and Development, v. 11, n. 1, e15311123711, 2022

(CC BY 4.0) | ISSN 2525-3409 | DOI: http://dx.doi.org/10.33448/rsd-v11i1.23711

Santos, C. H. (2017). Eficácia da terapia cognitiva processual e da ativação comportamental no tratamento do transtorno depressivo maior: um ensaio clínico randomizado. Tese de Doutorado em Processos Interativos dos Órgãos e Sistemas, do Instituto de Ciências da Saúde, Universidade Federal da Bahia, Salvador.

Rother, E. T. Revisão sistemática x revisão narrativa. Acta Paul Enferm. http: //www.scielo.Br/scielo.php? script= sci_arttext\&pid +S010321002007000200001

Rufino, S., Leite, R. S., Freschi, L., Venturelli, V. K., Oliveira, E. S. O, \& Mastrorocco Filho, D. A. M. (2018). Aspectos gerais, sintomas e diagnóstico da depressão. Revista saúde em foco, (10), 841.

Stein, A. T., Carl, E., Cuijpers, P., Karyotaki, E., \& Smits, J. A. (2021). Looking beyond depression: A meta-analysis of the effect of behavioral activation on depression, anxiety, and activation. Psychological Medicine, 51(9), 1491-1504.

Schatzberg, A. F. Cole, J. O., \& Battista, C. (2009). Manual de psicofarmacologia clínica. (6a ed) Artmed, 719 p.

Wright, J. H., Basco, M. R., \& Thase, M. E. (2008). Aprendendo a terapia cognitivo-comportamental: um guia ilustrado. Artmed. 